\title{
LA POLITICA DELLA SECESSIONE
}

\author{
di Gaspare Nevola
}

Un nuovo termine sta facendo irruzione nel nostro lessico politico corrente: secessione. E con esso, un nuovo fantasma s'aggira anche per l'Italia. Dopo e accanto alla questione settentrionale, al federalismo e al problema del senso di nazione, con la parola d'ordine «secessione» è ancora una volta la Lega a ridefinire l'arena del dibattito politico, introducendovi temi di riflessione inediti o trascurati nel nostro paese. Suo malgrado, essendo di altra natura i suoi intenti, il leghismo ci costringe ad affrontare importanti questioni politico-culturali. A dispetto del dubbio spessore della cultura politica della sua leadership, con le sue iniziative ad effetto, spregiudicate e talora preoccupanti, il leghismo sta certamente contribuendo ad un progressivo rifacimento del linguaggio politico di casa nostra. In particolare, con i proclami secessionisti del leghismo «padano» emerge ancora una volta la centralità della dimensione territoriale in politica. Siamo insomma di fronte ad un tipico problema di «politicizzazione del territorio». Ma siamo altresì collocati sul terreno della politica: della «politica della secessione».

Cì̀ detto, in questa sede non mi occuperò direttamente né del leghismo né della condotta politica e del secessionismo della sua leadership ${ }^{1}$. Mi propongo piuttosto di «prendere sul serio» le «uscite» secessioniste del leghismo ovvero di capire il fenomeno politico che in questo modo acquista corpo. Mentre mi sembra doveroso che ciascuno prenda posizione di fronte ad una questione, quale quella della secessione, che solleva la prospettiva della divisione dell'Italia come Stato-nazione, di seguito mi muoverò sul versante dell'analisi politica - sia normativa che esplicativa.

1 Sul caso italiano cfr. Rusconi (1994); Cartocci (1994); Diamanti (1995; 1996a; 1996b); Rampini (1996); AA.VV. (1996); Biorcio (1997); Rumiz (1997).

RIVISTA ITALIANA DI SCIENZA POLITICA / a. XXVIII, n. 1, aprile 1998 
La secessione rappresenta una sfida politica, ma anche una sfida concettuale all'analisi politica - specie in un contesto quale quello italiano, perlomeno estraneo ad istanze secessioniste e da più lungo tempo ancora poco preparato quanto a strumenti per analizzarle. Ė inutile negare che il sapere sociopolitologico non dispone di una vera e propria teoria generale (normativa ed esplicativa) della secessione. Al momento si danno dei frammenti, anche importanti ma ancora insoddisfacenti. L'obiettivo, in questa sede, non è di colmare in un colpo lacune di tale portata, quanto di recuperare qualche frammento di teoria della secessione e di provare ad elaborarne qualche altro in vista di un primo quadro di riferimento per l'analisi. Dunque, non mi occuperò direttamente del leghismo «padano» e della sua offensiva nel nostro paese, bensì del fenomeno politico della secessione in senso generale. $\dot{E}$ evidente che il quadro di riferimento analitico qui abbozzato potrà essere utilizzato per gettare luce anche sul caso italiano. Che poi quest'ultimo possa, più o meno velocemente, «sgonfiarsi», meglio ancora. Intanto resterà utile l'intento di arricchire la strumentazione dell'analisi politica di fronte alla problematica della secessione. Una problematica comunque ben viva in diverse parti del mondo, che costringe a ritornare su nodi cruciali della vita politica, troppo spesso dati per scontati, e a riflettere sui concetti che possono chiarirli.

\section{Lo statuto politico della secessione}

Nel suo significato etimologico derivato dal latino seceděre, «secessione» vuol dire «separazione», ed indica l'atto con cui un soggetto (un individuo o, soprattutto, un gruppo) defeziona, si ritira da una collettività più ampia di cui fa parte, ossia si divide da essa, decidendo di abbandonarla. In questa accezione generica, la secessione può essere equiparata all'opzione «uscita», la quale, secondo il noto schema di Hirschman (1982), insieme alla protesta e alla lealtà, dà luogo a modalità di azione dirette a porre rimedio al cattivo funzionamento di un'organizzazione sociale - si tratti di un'azienda in campo economico, di un partito o uno Stato in ambito politico.

In prima istanza, quindi, la secessione potrebbe essere intesa come una forma particolare di espressione di quel fenomeno più generale che è la «divisione». In altre parole, il riconoscimento dei valori della libertà e del pluralismo porta con sé la 
possibilità che i membri di una collettività si dividano, anche fino a contrapporsi, gli uni verso gli altri. È questo, del resto, il lascito storico del processo di costruzione dei sistemi politici moderni ${ }^{2}$, imperniati su linee di divisione (cleavages); ed a questo rimanda il liberalismo come «arte della separazione» ${ }^{3}$.

Tuttavia questa caratterizzazione non rende giustizia alla radicalità della secessione come fenomeno politico. La secessione e i movimenti che la reclamano ${ }^{4}$ non riguardano una rivendicazione qualunque, una rivendicazione politica ordinaria, rubricabile pianamente sotto i principi del pluralismo e della libertà. $\mathrm{Al}$ contrario, la secessione è qualcosa di molto radicale. Anzi: di massima radicalità. In questo essa ha qualcosa in comune con la rivoluzione e con la guerra civile: l'attacco al principio di legittimità che vincola la comunità politica all'interno della quale nasce l'istanza secessionista. Pur avendo origine nell'ambito di una data comunità politica organizzata, la secessione implica una sfida all'ordinamento di autorità costituito - tipicamente, nel mondo moderno, lo Stato-nazione. Secedere significa fuoriuscire da questo ordinamento. Mentre altre forme di espressione della divisione politica, ad esempio la ribellione o la protesta, tendono normalmente a configurarsi come rivendicazioni ordinarie che si collocano all'interno di un dato ordinamento di autorità (Gamson 1968; Gurr 1970; Nordingler 1972; Melucci 1976), la secessione è una prospettiva eccezionale proprio in quanto si proietta all'esterno dell'ordinamento costituito. E singolare, ma non casuale, allora, il fatto che Hans Kelsen (1994, 235) chiami «rivoluzione» quel processo attraverso il quale «una parte della popolazione di uno Stato si stacca e costituisce un nuovo Stato nel territorio in cui vive» - e cioè la secessione. Tutto ciò spiega l'«incubo dell'uscita» (Finer 1974) che da sempre angoscia lo Stato-nazione nato con la politica moderna, specie nell'Europa continentale - là dove uscita sta, chiaramente, per proclamazione di indipendenza territoriale o secessione.

2 Non a caso Dahl (1986) fissa nella liberalizzazione o legittimazione dell'opposizione uno dei processi costitutivi della costruzione dei sistemi democratici.

3 Sul primo aspetto cfr. Rokkan (1982), e prima ancora Hume (1987), sebbene Hume giudichi negativamente la «divisione partitica». Sul secondo aspetto Walzer (1984), e prima ancora Montesquieu (1989).

4 Inquadramenti concettuali e panoramiche dei movimenti separatisti sono rinvenibili in particolare nella letteratura dedicata alla questione etnica. Cfr. ad esempio Smith (1984); Horowitz (1985); Melucci e Diani (1992); Connor (1995); Toso (1996); Buttino e Rutto (1997). Una classificazione dei casi di secessionismo nel mondo contemporaneo è in Bookman (1993). 
In base a queste brevi considerazioni si può affermare che la secessione ha uno statuto squisitamente (anche se non esclusivamente) politico. Si tratta di un punto cruciale per capire la secessione. Un punto peraltro controverso. Spendiamoci qualche parola.

Secondo un orientamento molto diffuso, il principio di auto-determinazione dei popoli, che spesso è posto alla base della secessione, si colloca su un piano strettamente culturale: in questione sarebbe il diritto all'identità culturale di un gruppo, ossia la possibilità che questo possa affermare e tutelare i suoi specifici caratteri linguistici, religiosi, le sue tradizioni ${ }^{5}$. Questo orientamento, tuttavia, non convince. E non tanto perché sottolinea l'importanza della dimensione culturale, sulla quale non vi è nulla da eccepire. La debolezza di questa posizione consiste piuttosto nel tradurre la secessione in problema di tolleranza e di pluralismo culturale, per così dire, ordinario. Lasciamo al momento da parte la questione che l'istanza di secessione si nutre di ragioni anche utilitaristico-economiche, e non solo identitario-culturali. Resta il fatto che l'appello all'auto-determinazione, così come la secessione in generale, è fatto in nome di una pretesa a «decidere per conto proprio» sulle cose da fare, e innanzitutto sulla «cosa pubblica», ossia sugli aspetti pubblici riguardanti la vita di ciascuno. La posta in gioco è, insomma, l'autonomia e l'autogoverno, in virtù dei quali una collettività pretende di decidere anche (ma non solo) sui suoi aspetti culturali. La collettività, cioè, non si limita a chiedere riconoscimento, tolleranza o pari dignità per la propria specificità culturale, ma vuole gestire questa in modo sovrano - alla stregua di uno Stato nazionale nel consorzio internazionale. Se così non fosse, non si capirebbe come mai, ad esempio, i quebecois, che godono del riconoscimento e della tutela della loro lingua e della loro cultura da parte dello Stato federale canadese, continuano a considerare la loro come una condizione che richiede l'auto-determinazione ${ }^{6}$.

5 Cfr. ad esempio Nielsen (1993); Tamir (1993). Ma anche Taylor (1993) (con commenti di S. Wolf, S.C. Rockfeller, M. Walzer).

6 Cfr. De-Shalit (1996). De-Shalit critica la lettura «culturalista» della secessione/ autodeterminazione, contrapponendo ad essa quella politica. La strada sulla quale egli si pone è condivisibile, anche se la sua messa a fuoco dello statuto politico della secessione (o dell'autodeterminazione cui egli si riferisce) appare ancora difettosa. Sul caso del Quebec, considerato per molti aspetti paradigmatico del secessionismo contemporaneo nel mondo occidentale, esiste una mole di studi ormai imponente. Qui mi limito a ricordare solamente un recente dossier in «Limes»: AA.VV. (1995). 
Ma cosa intendiamo dire quando parliamo di statuto politico della secessione? Definiamo, intanto, la politica (o il «politico» come direbbe Carl Schmitt) come governo dell'ostilità tra gli uomini. «Governo» sta per arte della costruzione del consenso e «ostilità» per l'irriducibile pluralismo e conflittualità dei valori e degli interessi: la politica è, allo stesso tempo, confliggere e governare i conflitti (Nevola 1994). In senso radicale, politica è anche il luogo dell'agire in cui si decide dell'unità o della disunità di una comunità ${ }^{7}$. È politica in senso forte, allora, quell'azione con la quale si persegue l'uno o l'altro obiettivo. Come è evidente, questo è il caso della secessione, che si svela in tutta la sua radicale politicità.

Detto questo, bisognerebbe qualificare meglio lo statuto politico della secessione. Cosa non facile, vista anche una certa arretratezza degli studi in materia. E tuttavia qualche spunto va lanciato. La secessione scuote dalle fondamenta la costruzione politica dello Stato, la stessa unità, cioè, di una comunità politica organizzata in senso moderno ${ }^{8}$. Ciò perché implica una sfida alla sovranità dello Stato e del suo potere di decidere erga om$n e{ }^{9}$, una sfida che non può essere ricomposta all'interno dell'ordinamento di autorità costituito, dato che si pone «a monte della Costituzione e di ogni ordinamento» ${ }^{10}$.

Veniamo dunque agli attributi principali della sovranità ${ }^{11}$. Normalmente un'unità politica (ad esempio uno Stato, indipendentemente dalla sua forma e dalla sua forma di governo) è considerata sovrana quando pretende per sé ed è in grado di

7 Questa visione della politica deve non poco alla concezione che Carl Schmitt ha codificato in termini di «amico/nemico», sebbene non vi si identifichi del tutto quanto ad implicazioni e sviluppi. Cfr. Schmitt (1972). Per una ripresa della concezione schmittiana cfr. Freund (1983; 1995).

8 Per una discussione dei caratteri speciali dello Stato rispetto ad altre forme di associazione cfr. ad esempio Lasswell e Kaplan (1979, 193-208). Più in generale si veda Matteucci (1993).

9 Questo aspetto è stato sottolineato di recente anche in Italia. Cfr. Miglio e Barbera (1997); De Fiores e Petrosino (1996).

10 Così Miglio in Miglio e Barbera $(1997,180)$.

11 Più in generale, per una recente ricognizione storico-concettuale sul tema della sovranità cfr. Ferrajoli (1997). L'autore, in particolare, insiste sulle tendenze al venir meno delle ragioni della sovranità dello Stato-nazione nel mondo contemporaneo. Quanto più questa tesi dovesse rivelarsi fondata, sia sul piano normativo che su quello empirico, tanto più avremo un allargamento degli orizzonti (normativi ed empirici) del secessionismo - e non solo, come invece ritiene Ferrajoli, un affermarsi di tendenze integrative sovra-statali (e sovra-nazionali). Vedi anche Cassese (1984); Matteucci (1993); Bonanate (1994). 
definire, in linea di principio ed in larga misura: 1) l'unità e l'integrità territoriale (territorio, confini); 2) la gestione degli interessi pubblici e la ridistribuzione delle risorse (moneta, tassazione); 3) l'imposizione del comando via autorità (leggi, monopolio della forza fisica); 4) i criteri di inclusione nella ed esclusione dalla comunità stessa (appartenenza nazionale, popolo, cittadinanza). La secessione mette in discussione tutti e quattro questi elementi della sovranità e con essi la sovranità stessa. Così, per parafrasare Schmitt, si potrebbe dire che «sovrano è chi decide sulla secessione» (intesa come «stato di eccezione») ossia chi potrà disporre del territorio, delle risorse pubbliche, dell'imposizione del comando, della definizione dell'appartenenza nazionale o cittadinanza. Più avanti ritornerò sui primi due elementi, dato che le formulazioni prevalenti del diritto di secessione chiamano in causa in particolare la questione del territorio e quella della distribuzione pubblica delle risorse. Al momento mi soffermo brevemente sugli ultimi due aspetti, non poco trascurati ed invece fondamentali. E soprattutto di fronte ad essi che si qualifica meglio lo statuto (radicalmente) politico della secessione.

La sovranità di uno Stato, sappiamo, non può basarsi sempre ed esclusivamente sull'esercizio o minaccia della forza. Anzi: essa è propriamente tale se si accompagna alla legittimità, di qualunque tipo essa sia - la lezione weberiana a questo riguardo resta ferma. È perciò essenziale che non si interrompa il processo di legittimazione, sul quale in ultimo si fonda una comunità politica e la sua stabilità, anche in caso di conflitto. La dottrina democratica parla a questo proposito di patto/compromesso sulle regole, condiviso dalle parti. Al fondo di tutto, $\mathrm{i}$ teorici del realismo politico collocano, invece, l'arcano dell'«obbedienza civile» - e cioè la «misteriosa» circostanza in base alla quale alcuni uomini obbediscono ad altri uomini. $\mathrm{Ma}$ al di là di questo, qui è importante sottolineare che il processo di legittimazione è alla base dell'autorità e dell'identità di un gruppo costituito come comunità politica, così come è a fondamento della stabilità e continuità di questa. La legittimazione è essenzialmente un processo di «riconoscimento ed accettazione» dell'allocazione dell'autorità (Stato) e dei confini dell'appartenenza (nazione). Il principio di legittimazione pone che $\mathrm{i}$ membri di una comunità politica sovrana: 1) operino nell'obbligo del rispetto della catena di comando e delle decisioni vincolanti prodotte dall'autorità; 2) si riconoscano come tali (e cioè 
come appartenenti a una comunità politica) e come soggetti per i quali valgono le decisioni vincolanti «autorizzate» da essi stessi come collettività.

A fronte di tutto questo, la secessione implica la fuoriuscita dai vincoli dell'autorità e dell'appartenenza. Quando un gruppo vuole staccarsi dalla comunità statale-nazionale di cui (a torto o a ragione) fa parte, di fatto esso afferma di non riconoscere (più) come validi ed obbliganti, da un lato, i comandi derivanti dalla catena di autorità costituita; dall'altro, i criteri, i confini e i vincoli dell'appartenenza. Con la secessione non viene più accettata quella definizione che stabilisce «chi siamo» e «a chi obbediamo». Quando la secessione è chiamata in causa in uno Stato nazionale sovrano e democratico, come oggi capita con insolita frequenza, anche in Italia, la questione diviene particolarmente delicata. Viene infatti ad essere investito quel principio di legittimità in base al quale le pretese di autorità e di appartenenza dello Stato-nazione devono essere riconosciute come valide e, appunto, legittime. Riconosciute da coloro che sono toccati da tali pretese. Il principio di legittimità unifica una comunità politica. $\mathrm{Ma}$ è sul principio di legittimità che si consuma altresì la sua divisione secondo forme non ordinarie o funzionali (riguardanti ad esempio specifiche competenze, prerogative), ma secondo quella forma radicale che riguarda la sovranità stessa.

Ora, a dispetto di sue razionalizzazioni amministrative, legali o costituzionali ${ }^{12}$, in ultimo il principio di legittimità resta un principio squisitamente politico proprio perché politica è la sfera in cui si decide dell'unità o meno di una comunità e del suo ordinamento sovrano. Il caso della secessione, prima ancora che come separazione territoriale, va definito come abbandono dei vincoli dell'autorità suprema (sovranità statale) e dell'appartenenza (identità e cittadinanza nazionale). Nel caso della secessione, le contese e $\mathrm{i}$ conflitti spingono la politica fuori dal terreno ordinario del potere costituito (Stato-nazione sovrano), verso il terreno «eccezionale» del potere costituente, dove si lotta per il potere politico e per l'identità politica con pretese di legittimità che le parti si contestano reciprocamente ${ }^{13}$. E questo che

12 Principi di legittimità così concepiti sono sostenuti, ad esempio, da Luhmann (1969); Habermas (1996); Zagrebelsky (1992). Simili concezioni affondano le loro radici nelle dottrine del costituzionalismo moderno, da Kelsen (1994) a Mcllwain (1990), per citare solo due notevoli esponenti.

.13 E in contesti di questo tipo che Tilly (1978) richiama il concetto di «sovranità multipla». Cfr. anche Nevola (1994). 
segna lo statuto radicalmente politico della secessione come sfida al livello supremo del principio di legittimità - in analogia con quanto avviene con i casi della rivoluzione e della guerra civile.

\section{Diritto di secessione}

Il fatto che la secessione abbia uno statuto politico, in quanto pone sfide sul piano della legittimità di un ordinamento di autorità costituito, è un aspetto qualificante ed importante che si tende talora a trascurare o minimizzare. $\mathrm{Ma}$ ciò non dice ancora nulla sulla legittimità della secessione stessa. $\mathrm{O}$ meglio: solleva il problema della legittimità delle rivendicazioni secessioniste. Questo problema è innanzitutto di natura normativa. E costituisce l'aspetto più trattato nel dibattito internazionale che di tanto in tanto affiora - nel quale, non a caso, protagonisti principali sono gli studiosi di filosofia politica (Bucheit 1978; Beran 1984 e 1988; Birch 1984; Brilmayer 1991; Buchanan 1994 e 1997; Twining 1991; Kymlicka 1995). La secessione come questione normativa apre così il capitolo degli argomenti sulla sua giustificabilità o meno. La secessione diventa un'istanza $o$ un'azione politica legittima nella misura in cui trova giustificazione, ossia se viene riconosciuta come ammissibile intanto in linea di principio - esattamente come avviene per ogni altra istanza o azione in campo politico. Tipicamente, i fondamenti di legittimità normativa vanno ricercati nell'ambito del discorso morale, cui compete la determinazione di ciò che può o non può essere ritenuto giusto ${ }^{14}$. Dunque: è moralmente giusto reclamare e perseguire la secessione? Nel linguaggio della filosofia politica anglosassone: è dato un «diritto morale» alla secessione? $\mathrm{E}$ in particolare, un tale diritto è affermabile in democrazia?

Lasciamo al momento da parte la questione di «chi decide» o definisce come moralmente giusta e legittima la secessione questione che inesorabilmente sposterebbe da subito l'attenzione dal discorso morale all'analisi politico-fattuale. Consideriamo invece cosa comporta, in generale ed in linea di principio, trat-

14 Interessanti punti di vista su quella problematica classica concernente il ruolo del discorso morale nell'analisi dei fenomeni sociali e politici sono, tra altri, Walzer (1987; 1990a); Habermas (1985; 1996); Hampshire (1995); Berger (1981). 
tare con argomenti di moralità la legittimità (presunta o pretesa) della secessione.

Immettersi su questa strada significa, innanzitutto, ammettere la possibilità che le istanze di secessione siano riconosciute come legittime sul piano morale. Considerare la secessione in termini di eversione diventa allora solo uno dei modi in cui è possibile definire il fenomeno. Per essere precisi, è il modo tipico in cui i difensori dell'ordinamento statuale-nazionale definiscono la secessione. Ma una definizione del genere è niente affatto aproblematica. Anzi: risulta irta di difficoltà nell'ambito della stessa dottrina liberaldemocratica (Buchanan 1994; Kymlicka 1995), la quale, ricordiamolo, ha tradizionalmente speso argomenti a sostegno della legittimità (naturalmente condizionata) della disobbedienza civile di fronte al potere costituito dello Stato $^{15}$. Ciò considerato, è evidente che parlare di legittimità morale della secessione, e quindi riconoscere in linea di principio un diritto di secessione, significa fare un'affermazione già di per sé molto impegnativa, niente affatto scontata nella cultura politica dominante ad esempio nel nostro paese - come si può desumere dal dibattito pubblicistico, il quale raramente si cimenta con una seria considerazione del problema della secessione, impegnato, quando lo è, a stigmatizzare le «uscite» secessioniste del leghismo, le sue minacce all'unità della nazione e con ciò a segnare l'illegittimità (quasi per definizione) della secessione. Diversamente, nel dibattito internazionale la tesi che la secessione abbia una sua legittimità morale rappresenta la posizione prevalente tra gli studiosi della secessione che si muovono sul terreno normativo. I quali, nella maggior parte dei casi, concludono in positivo sulla legittimità morale della secessione, sebbene con la sottolineatura di limitazioni e condizioni vincolanti in generale e caso per caso (Bucheit 1978; Beran 1984; Birch 1984; Kymlicka 1995). Sarebbe sintomo di superficialità o

15 Al riguardo, un riferimento classico nella pubblicistica politica è Thoreau (1993). Nello stesso volume si veda anche il saggio di Miglio (1993). Esiste naturalmente un'imponente e secolare letteratura dottrinaria e pubblicistica sulla disobbedienza civile (come anche sul «diritto di resistenza»). Un contributo stimolante, calato in un momento «caldo» come quello a cavallo tra gli anni ' 60 e '70, è Arendt (1985), dove si legge, significativamente: «Forse ci voleva una situazione di emergenza prima che potessimo trovare un posto adeguato alla disobbedienza civile, non solo nel nostro linguaggio politico, ma anche nel nostro sistema politico. E si è sicuramente di fronte ad una situazione di emergenza quando i poteri costituiti di un paese non riescono a funzionare adeguatamente e quando la loro autorità non ha più forza» (p. 160). 
di arroganza etico-politica e culturale ritenere che si possa prendere sul serio il fenomeno del secessionismo senza fare $\mathrm{i}$ conti anche su questo terreno. D'altra parte dobbiamo però prendere atto che, come è stato osservato, «disporre di un diritto morale significa godere di un potere o di un'autorità particolarmente forte e, di conseguenza, l'obbligo da parte di altri di non interferire nelle attività di chi esercita un proprio diritto è un obbligo particolarmente vincolante» (Buchanan 1994, 53). Da qui una duplice rilevanza, già in linea di principio, del diritto morale alla secessione: una rilevanza etico-politica, poiché esso fonda le «buone e giuste» ragioni di una rivendicazione; ed una rilevanza strategico-politica, poiché esso fornisce un'utile arma di persuasione/coercizione nel conflitto secessionista. Insomma, pretendere e riconoscere un diritto, ancorché morale, resta una cosa seria ${ }^{16}$.

Affrontando la secessione da questa angolatura di analisi, il primo aspetto che va fermato è il riconoscimento di quello che viene chiamato il «diritto di secessione». Un diritto che si qualifica innanzitutto nell'ambito del discorso morale cui compete la regolazione del giusto e dell'ingiusto, del lecito o dell'illecito nella sfera dell'azione politica. Dunque un diritto, per così dire, pre-giuridico e pre-costituzionale, se vogliamo fondativo del diritto propriamente giuridico-costituzionale, ma non del tutto coincidente con quest'ultimo ${ }^{17}$. Insomma, un diritto morale che in quanto tale resta fuori dalla cornice normativa definita dal diritto positivo moderno, quello incarnato in ordinamenti costituzionali, leggi e norme giuridiche, codici autoritativi, rispondenti al principio dell'effettualità del diritto e dotati di strumenti di sanzione giuridica per la loro applicazione.

Il diritto di secessione, nel senso sopra inteso, trova intanto riconoscimento di principio nel quadro dei valori etico-politici della civiltà occidentale e, più precisamente, della cultura politica della modernità. La trasformazione dei sudditi in cittadini, il passaggio all'età dei diritti, l'inclusione delle masse nel processo

16 Rimane naturalmente tutto da discutere il tema riguardante l'esistenza di una relazione (nel caso sicuramente complessa) tra diritti morali e diritti giuridici, i quali, nel caso della secessione, trovano (o troverebbero) collocazione in sede di diritto costituzionale, di diritto internazionale e, per taluni aspetti, anche nel diritto penale. Sul piano del diritto costituzionale cfr. Buchanan (1994); su quello del diritto internazionale Michalska (1991); Bhalla (1991).

17 Per la distinzione tra diritto in senso giuridico e diritto in senso morale (tipica nella cultura politica anglo-americana) cfr. ad esempio Walzer (1990a). 
di decisione politica (voto, reperimento del consenso e di legittimazione), il riconoscimento della libertà hanno segnato la nascita di un nuovo universo politico imperniato sul principio dell'autogoverno da parte delle comunità politiche ${ }^{18}$. In questo quadro di valori, quindi, detto in termini essenziali, il principio di autogoverno pone le premesse normative del diritto/pretesa della secessione come opzione nell'arena politica ${ }^{19}$. Naturalmente, un simile riconoscimento, per quanto di per sé cruciale, non è sufficiente per un discorso normativo sulla secessione. Da un lato dice molto, poiché definisce la secessione un'opzione legittima sul piano etico-politico, frantumando per ciò stesso le mura di cinta ideologiche dell'indivisibilità e dell'unità poste a difesa dello Stato-nazione. Dall'altro lato, tuttavia, dice troppo poco, dato che risultano ancora inespressi ed inarticolati gli argomenti morali che possono, per così dire, espugnare sul piano normativo lo Stato-nazione, fino a provocarne uno spezzettamento, una divisione. Infatti, una volta infrante le sue mura perimetrali, lo Stato-nazione diventa normativamente attaccabile, ma resta ancora una «fortezza normativa», con solidi sistemi di difesa che vanno superati prima che la si possa normativamente espugnare. Per questo, in un discorso normativo sulla secessione vanno messe in luce le armi di attacco e di difesa (normativa) della fortezza-Stato nazionale. Queste armi sono gli argomenti morali, con i quali si individuano le «ragioni» che giustificano la secessione, ma anche quelle che ne sanciscono l'illegittimità morale.

Nel dibattito normativo internazionale circolano differenti orientamenti e proposte ${ }^{20}$. Sebbene l'insieme di tali argomenti condivida un medesimo statuto morale, se guardiamo alla natu-

18 È alla luce del principio dell'autogoverno democratico che, ad esempio, Alexis de Tocqueville legge la rivoluzione americana e gli assetti statali federalistici della nazione americana e li confronta con l'ancien régime del vecchio continente europeo. $\mathrm{Cfr}$. Tocqueville (1968).

19 Cfr. ad esempio Buchanan (1994); Kymlicka (1995). In questa chiave va letta, ad esempio, anche una considerazione classica, come la seguente di John Stuart Mill: «Il problema del governo va... deciso dai governati. Una collettività deve poter essere libera di associarsi come meglio desidera»; di conseguenza, «sarebbe utile e anche necessario spezzare ogni legame politico quando le nazionalità non possono fondersi» (Stuart Mill 1997, 218 e 223).

20 Tra i principali schemi di argomenti morali pro e contro la secessione particolarmente articolato e discusso nei dettagli è quello fornito in Buchanan (1994). Ma si vedano anche gli schemi più compatti proposti, ad esempio, in Beran (1984); Birch (1984); Brilmayer (1991). 
ra specifica dei motivi chiamati in causa, essi possono essere ripartiti, per grandi linee, in alcune categorie principali. Una prima categoria comprende argomenti di contenuto «culturale», quali la salvaguardia delle culture, l'accrescimento della diversità culturale, il principio di nazionalità (Buchanan 1994; Nielsen 1993; Tamir 1993; Kymlicka 1995). A suo modo, questo tipo di argomenti morali sembrerebbe quello maggiormente fondativo del diritto/pretesa di un gruppo alla secessione, visto che si basa su quel criterio di identità collettiva (culturale) che definisce i caratteri specifici di un gruppo (lingua, religione, patrimonio artistico-letterario, tradizioni, usi e costumi) e il senso di appartenenza che lega i membri di un gruppo tra loro e al gruppo stesso. Tuttavia, come questi motivi - da soli - non sono sempre sufficienti a definire l'identità collettiva di una comunità (come sappiamo, ad esempio, nel caso di quella comunità che è la nazione $)^{21}$, così essi non sono considerati tra $i$ più probanti della legittimità morale della secessione. D'altra parte, la categoria di argomenti morali pro-secessione più ricca e mai trascurata nel dibattito internazionale è un'altra: quella che si riferisce a motivi di contenuto «politico» (e «politico-territoriale»). In questo caso si parla di principio del consenso popolare e regola di maggioranza, di salvaguardia della libertà e dei diritti ed autodifesa o protezione fisica di un gruppo, fino a richiamare $\mathrm{i}$ principi di autodeterminazione dei popoli e della sovranità - e cioè i pilastri fondativi di ogni ordine politico costituito (Beran 1984; Birch 1984; Brilmayer 1991; Buchanan 1994; Kymlicka 1995; De-Shalit 1996). Una terza categoria di argomenti morali pro-secessione ha come contenuto motivi di ordine storico, quali l'inclusione di una comunità, di una regione nell'ordinamento e nel territorio statale-nazionale perpetrata con la forza oppure l'istanza di rettificare ingiustizie subite nel passato (Birch 1984; Buchanan 1994). Infine, in una quarta categoria possiamo includere quegli argomenti che rimandano a motivazioni di tipo economico - che spesso incontrano forte presa presso la base sociale del secessionismo, sebbene non sempre risultino decisivi come a tutta prima potrebbe sembrare. La tutela di interessi economici di una regione, l'esigenza di sottrarsi ad una

21 Il dibattito sulla nazione in questi ultimi anni si è riacceso anche in Italia, sia in sede scientifica che in sede pubblicistica. Punto di riferimento è Rusconi $(1993 ; 1997)$. Per un primo inquadramento della problematica e per riferimenti bibliografici più puntuali mi limito a rimandare a Cuaz (1997); Nevola (1997). 
ridistribuzione discriminatoria delle risorse economiche e la prospettiva di aumentare l'efficienza economica, commerciale e produttiva illustrano bene quest'ultima categoria di argomenti (Birch 1984; Buchanan 1994; Bookman 1993).

$\grave{E}$ appena il caso di accennare che accanto a questi argomenti morali pro-secessione stanno quelli anti-secessione, i quali si ripartiscono, nel complesso, nelle stesse categorie in cui abbiamo dislocato i primi e che, in non pochi casi, di fatto si riferiscono agli stessi problemi toccati dalle motivazioni secessioniste, ma ovviamente con valutazioni ed argomentazioni di segno opposto. Così come mi limito appena ad esplicitare che i vari argomenti morali pro e anti secessione richiedono di essere, di volta in volta, circostanziati ed approfonditi alla luce delle specifiche situazioni concrete. $\mathrm{Da}$ una disamina di questo tipo, sulla quale non posso qui soffermarmi, discende la possibilità di considerare ciascun argomento morale in termini di condizione necessaria elo sufficiente per la legittimità morale di una data istanza secessionista, ma anche per la sua eventuale illegittimità.

Ciò detto, non mi soffermo ulteriormente e in dettaglio sugli argomenti morali a favore o contro la secessione. Eccetto una breve sottolineatura che mi pare interessante fare fin da subito, data l'opinione diffusa nel nostro paese. Mi riferisco all'argomento morale su base di considerazioni economiche. Ebbene, questo argomento, nel dibattito internazionale, è finalizzato a fornire una giustificazione della secessione anche in vista della tutela di interessi (materiali) egoistici o particolaristici di un dato gruppo, senza lasciarsi distogliere dal fatto che la secessione sia voluta dalla parte ricca di uno Stato-nazione e che ne accresca i vantaggi economici a scapito della parte restante e più povera (Buchanan 1994; più in generale Bookman 1993). Come è facile capire, questo aspetto è di particolare attualità anche in Italia, di fronte alla specifica fisionomia che va assumendo la secessione pretesa dal leghismo «padano», dove inquietudini, paure ed aspettative economiche e socio-territoriali che percorrono il nostro «nord-est» si intrecciano con la condotta politica del leader della Lega Nord.

Senza entrare nel merito della vicenda di casa nostra, anche nel caso dell'argomento morale di contenuto economico, esattamente come gli altri, l'istanza secessionista va calata sul terreno specifico degli argomenti morali favorevoli o contrari, situazione per situazione. Il suo riconoscimento in termini di diritto morale non consente di respingerla in linea di principio, ma 
non permette nemmeno di accoglierla in assoluto. Essa apre, piuttosto, alla possibilità e al dovere morale di sottoporla al banco di prova degli argomenti morali: questi diventano il test e il filtro della secessione come diritto condizionato.

Una considerazione di fondo imprescindibile è, pertanto, che gli argomenti morali prospettano, alla fine, una concezione della legittimità condizionata della secessione. Ossia essi inducono, da un lato, a discutere quali possano o debbano essere le condizioni che giustificano le istanze a secedere e quali quelle che le rendono moralmente irreclamabili; dall'altro, a verificare, caso per caso, se si danno o meno quali condizioni. Ad emergere, in molte circostanze, è il carattere controverso o ambivalente, e talora non risolutivo, di numerosi argomenti morali, sia pro sia anti secessione - soprattutto nei casi concreti. Al discorso normativo spetta così il compito di elaborare argomenti pro o anti secessione, dalla composizione e dalle caratteristiche dei quali potrà risultare il grado di permissività morale della secessione di una cultura politica comunque decisa a prendere sul serio la sfida secessionista anche sul piano normativo.

$\mathrm{Ma}$ anche un'altra acquisizione merita di essere tenuta ben in rilievo, per disporci ad un'adeguata comprensione del fenomeno politico della secessione: la natura variabile dei motivi che giustificano o condannano la secessione come diritto morale. Così, gli argomenti morali, di un segno o dell'altro, fanno riferimento a condizioni e a rivendicazioni che si collocano, volta a volta, nella sfera culturale, in quella politica (e territoriale), in quella storica, in quella economica. Si tratta di un punto sul quale avremo modo di ritornare.

Raccogliere seriamente la sfida secessionista sul piano normativo ha tuttavia altre importanti e complicate implicazioni, che non possono essere tralasciate in uno schema per l'analisi del fenomeno. Sopra si è accennato che nel dibattito normativo internazionale è ben evidente la tendenza ad assegnare un titolo morale alla secessione. Non si tratta, come è intuibile, di una tendenza indolore, che non solleva imbarazzi o repulse. Di fatto, anche chi non avesse o limitasse i pregiudizi antisecessionisti si imbatterebbe nel problema del «diritto» di secessione. Ciò perché questo diritto è di una categoria del tutto particolare: è un «diritto di gruppo» ${ }^{22}$. Come è noto, un diritto di questo tipo

22 Altri casi di diritto di gruppo sono, ad esempio, il diritto di annullamento e di 
non appartiene alla dottrina liberaldemocratica ${ }^{23}$. O meglio, fatica a trovare coerente collocazione in quella tradizione di pensiero etico-politico e giuridico che ha permeato la cultura della modernità e che ha costituito il principale tessuto ideologico dello Stato di diritto, ossia di uno dei pilastri principali dei sistemi democratici, fino ai nostri giorni. Il principio individualistico e i diritti individuali sono una conquista delle rivoluzioni liberali e democratiche, la cui finalità era di restituire dignità, eguaglianza, libertà e sovranità al singolo individuo, liberandolo dalle catene spersonalizzanti costituite dalle varie forme dei corpi intermedi e tutelandolo di fronte al potere dello Stato-sovrano. Basti pensare alla vicenda del diritto di voto, e all'importanza del riconoscimento del singolo individuo come soggetto titolare - un vero e proprio meccanismo ideologico ed istituzionale di rottura dei vincoli dello Stato cetuale e dell'ancien régime (Bendix 1969; Rosanvallon 1994). Le rivoluzioni democratiche hanno riplasmato i rapporti politici ed etico-giuridici tra Stato e (singoli) cittadini, sbaragliando il vecchio ordine politico incentrato sui corpi intermedi ${ }^{24}$. E su queste nuove basi è andata via via riconfigurandosi la costruzione dello Stato-nazione. Insom$\mathrm{ma}$, lo Stato-nazione nella sua versione liberaldemocratica ha finito col mettere fuori gioco forme di diritto di gruppo (o collettivo), a vantaggio del diritto individuale. Il punto è invero più sfaccettato di quanto possano dire questi secchi richiami, e meriterebbe ben altra attenzione, anche soltanto in riferimento al problema della secessione (Kymlicka 1995; e più in generale Taylor 1993). Ma dato che qui interessa costruire una mappa teorico-analitica del fenomeno della secessione nella molteplicità dei suoi aspetti, e dato che, pur nell'esiguità degli studi sulla secessione, le questioni di tipo normativo (quale è quella sulla controversia tra diritto di gruppo e diritti individuali) sono quelle maggiormente trattate, qui procedo oltre, ritenendo più utile serbare spazio per altre più trascurate.

Un aspetto, tuttavia, mi pare doveroso mettere in rilievo.

veto (previsto in costituzioni di tipo federale), il diritto di proprietà collettiva, i diritti linguistici di minoranza. Cfr. Buchanan (1994).

23 Con questo non sto sostenendo l'identificazione tra liberalismo e democrazia, né proponendo una semplificazione armoniosa del rapporto tra i due. Cfr. Sartori (1993). Restano però l'incontro e l'interpenetrazione tra le due dottrine e gli arrangiamenti politico-istituzionali che esse hanno promosso.

$24 \mathrm{Si}$ vedano le efficaci sintesi storico-concettuali di questo processo, predemocratico prima e democratico poi, in Schnur (1979) e Poggi (1978). 
Sul piano normativo, il riconoscimento del diritto di secessione in quanto diritto di gruppo provoca forti tensioni in una concezione liberal democratica. E chiaramente impensabile rinunciare ai diritti individuali legati alla tradizione liberaldemocratica. Né, d'altra parte, è cosa agevole rifiutare il diritto di secessione sulla base di principi liberaldemocratici.

In primo luogo, perché questi ultimi sono da qualche tempo (ma lo sono stati anche in passato) al centro di profonde critiche portate dalle dottrine comunitarie, che in alcuni casi pongono seriamente in difficoltà la dottrina liberale più intransigente per la sua incapacità a far fronte ai numerosi problemi che attraversano la società e la politica contemporanea, tutti situati in un modo o nell'altro su quei versanti dell'identità o dell'appartenenza collettiva che faticano a trovare posto in una visione liberale puramente individualistica ed utilitaristica della vita politica ${ }^{25}$. In secondo luogo, perché la stessa tradizione liberaldemocratica, dopotutto, col diritto all'autogoverno e all'autodeterminazione, riconosce a suo modo forme di diritto di gruppo. Non è casuale, allora, che il dibattito più interessante sul diritto di secessione diventa alla fine un «dibattito tra liberali» (Beran 1984; Birch 1984; Buchanan 1994; Kymlicka 1995).

Con ciò, tuttavia, ritengo che il rifiuto del diritto di secessione non dipenda dall'inammissibilità dei diritti di gruppo in quanto tali. Le ragioni stanno altrove. Altrove rispetto al diritto di gruppo. E altrove rispetto alla dottrina liberaldemocratica. $\grave{E}$ lo statuto politico della secessione che torna prepotentemente in causa, e con esso lo Stato-nazione. E difatti, senza rinunciare né al diritto di gruppo né ai diritti individuali, sul piano normativo non mancano le proposte di integrare il diritto di secessione nella dottrina liberaldemocratica (Kymlicka 1995; Buchanan 1994 e 1997; Beran 1984; Birch 1984; Taylor 1993; a suo modo Walzer 1991). Qui non importa se si debbano introdurre delle revisioni in quest'ultima, e se occorrano, nel caso, pochi o molti gradi di revisione. Certo è che il diritto di secessione dovrebbe comunque assicurare il riconoscimento dei diritti degli individui, senza annullare questi nella collettività. Un punto, questo, non poco controverso, sul quale va ad incidere la legittimità

25 Tra i diversi contributi su questo argomento cfr., ad esempio, Chapman e Shapiro (1993); Ferrara (1992) (con saggi, tra gli altri, di M.J. Sandel, A. MacIntyre, Ch. Taylor, C. Larmore, R. Dworkin). 
condizionata della secessione e il grado di permissività morale cui essa dà adito nei differenti sistemi di argomenti morali.

In ultimo, il diritto di secessione come diritto di gruppo può avvalersi di due orizzonti dottrinari: non solo quello comunitario, ma anche quello liberale. La tendenza più promettente appare comunque quella che cerca di coniugare insieme un comunitarismo soft con un liberalismo soft, laddove però aumenterà lo spazio per situazioni dilemmatiche e controverse di fronte alla tutela di un diritto di una collettività e, al contempo, dei diritti del singolo individuo. Sostenere che un individuo va tutelato in quanto individuo e che un individuo è tale in quanto membro di una collettività con sue proprie caratteristiche e specificità appare una buona impostazione sul piano dei principi ${ }^{26}$ : E tuttavia è una lezione per niente facile da realizzare nei casi concreti.

Chi è il gruppo che secede? La secessione tra attore collettivo $e$ comunità socio-territoriale

Il diritto di secessione in quanto diritto di gruppo solleva un'altra importante questione: chi è il gruppo, la collettività titolare di quello che è, appunto, un «diritto di gruppo»? A chi va riconosciuta la legittimità morale a secedere?

Si tratta di un interrogativo particolarmente intricato, che mette a dura prova il discorso normativo. Comunque si formulino i criteri della legittimità condizionata della secessione, restano infatti da identificare le unità collettive alle quali applicare tali criteri. È chiaro che si tratterà di sotto-unità di quell'ampia comunità politica che è lo Stato-nazione. Ma come circoscrivere queste sotto-unità? Come riconoscere a queste ultime quei requisiti di «identità collettiva» che costituisce il passaggio

26 È ad un quadro di questo tipo che fanno riferimento, ad esempio, la «teoria dei diritti misti» di Buchanan o la «teoria dei diritti differenziati-per-gruppo» di Kymlicka. Con la prima si sostiene che «i più importanti diritti individuali possono trovare giustificazione su basi 'non individualistiche', collettivistiche o comunitarie. Per esempio, i diritti di libertà d'espressione, di religione, di assemblea e di associazione, benché conferiti a individui, garantiscono la tutela dei gruppi e dei loro valori» (Buchanan 1994, 135). Con la seconda si sostiene una concezione sostanzialmente convergente con la prima, sebbene sulla base di una tipologia più articoltata e sofisticata dei diritti da riconoscere a minoranze nazionali (su basi etniche, linguistiche, religiose). Cfr. anche Taylor (1993); Walzer (1990b). 
obbligato affinché ciascuna di esse possa avvalersi del diritto (di gruppo) a secedere - e quindi a costituire una comunità (politica) sovrana e separata da quella statuale-nazionale più ampia di cui fa parte?

La risposta più diretta e apparentemente più semplice sta nell'identificare la comunità socio-territoriale come «nazione». A pretendere legittimamente sovranità ed indipendenza è la nazione - con i suoi elementi costitutivi (dalla lingua alla storia), patrimonio comune di un popolo in cerca di un «proprio» territorio. Così un gruppo che può rivendicare il diritto a secedere deve essere un gruppo che si qualifica come nazione - e come nazionalista ${ }^{27}$. Tuttavia, lo statuto estremamente problematico della nazione finisce col rendere la nostra questione più complicata anziché più semplice, tirandosi dietro l'interminabile e spinosa discussione in cui ci si chiede se le nazioni «esistono» o sono «invenzioni», se sono delle «costruzioni» o delle mere «finzioni». Il punto è di grande importanza, e probabilmente una riflessione congiunta su nazione e secessione aiuterebbe a chiarire non pochi nodi intricati riguardanti e l'una e l'altra tematica. Al momento, senza andare troppo lontano, resta però il fatto che chiamare in causa la nazione significa solo spostare in avanti i termini della questione. In altre parole, si cederebbe alla tentazione di riversare sulla nazione, sui suoi problemi e criteri di identificazione, le difficoltà che si incontrano con l'identificazione della comunità titolata a secedere. E non solo. Sappiamo, infatti, che il ricorso al criterio della nazione trova coerenti argomenti morali per una fondazione normativa della secessione, argomenti in parte di contenuto politico-territoriale (autodeterminazione, sovranità, territorio) e in parte di contenuto culturale (lingua, tradizioni culturali, religione, ecc.). E tuttavia tali argomenti non godono di alcuna particolare priorità negli orientamenti normativi sulla secessione, vengono considerati accanto ad altri. Anzi, talune posizioni di liberalismo radicale li considerano argomenti particolarmente controversi e perciò non decisivi per dirimere i problemi normativi inerenti le pretese secessioniste (Buchanan 1994).

Alle prese con l'identificazione del gruppo che secede-con-

27 Così è ad esempio per Connor (1995); Hechter (1979), e per tutti coloro che pongono l'accento sull'autodeterminazione (di un popolo) più che sulla secessione. In alternativa (o in sovrapposizione) all'identità collettiva-nazione, spesso viene richiamata l'etnia. 
diritto, la teoria della secessione incontra dunque una serie di problemi concettuali, $\mathrm{i}$ quali in un modo o nell'altro ruotano attorno al tema dell'«identità collettiva». Anche questo è un terreno in gran parte ancora da esplorare e che non può essere tenuto ai margini di un'analisi compiuta del fenomeno secessionista. Percorrerlo significa imbattersi nei grovigli e nella sfuggevolezza del concetto stesso di identità collettiva. Qui possiamo tenere solo sullo sfondo la rilevanza del capitolo dell'identità collettiva (in particolare in campo politico), senza aprirlo direttamente. Mi limito, invece, ad osservare che la risposta agli interrogativi posti in apertura di questo paragrafo va ricercata, intanto, lungo due direzioni.

La prima ci suggerisce di identificare sul piano socio-territoriale il gruppo per il quale si pone il problema di un diritto di secessione. Il riferimento al territorio risulta evidentemente saliente ${ }^{28}$, per il semplice fatto che la secessione implica la separazione di una parte di territorio da quel più ampio territorio sul quale si esercita la sovranità dello Stato-nazione. La dimensione sociale è parimenti imprescindibile se non si vuole ridurre il territorio a puro oggetto fisico, qualificato e quindi eventualmente circoscritto in base a caratteri squisitamente geografico-natura$\mathrm{li}^{29}$. E evidente che la rilevanza del fattore territoriale fa aggio sugli attributi sociologici di questo. Ciò che rileva è la dimensione sociale $o$, per così dire, la «costruzione sociale» di quell'unità territoriale per la quale è in questione la separazione. Abbiamo quindi a che fare con un «territorio con la 'sua' popolazione» o, meglio, con una «popolazione con il 'suo' territorio». L'identità collettiva alla quale riconoscere il diritto di secessione come diritto di gruppo e alla quale applicare gli argomenti morali pro e contro è, in questo caso, quella che negli studi sociologici viene fermata col concetto di «comunità». Nella fattispecie che ci interessa, particolarmente illuminante è quella definizione di comunità come «gruppo per il quale l'uso di un determinato territorio rappresenta un elemento fondamentale nei rapporti sociali esistenti fra i suoi membri» (Tilly 1976, 64). Questa definizione di tipo ecologico e piuttosto

28 La rilevanza del territorio in tema di secessione, sulla quale si registra ampio consenso, è particolarmente sottolineata in Brilmayer (1991).

29 Questa visione socio-territoriale è stata utilizzata in modo esemplare in due studi su altri argomenti ma a loro modo istruttivi anche per il nostro: Tilly (1976); Hechter (1979). 
estremistica valorizza ottimamente il territorio come fattore di identificazione delle sotto-unità per le quali può essere in questione la secessione, con un utile effetto di semplificazione nell'analisi, dato che non contempla di prestare attenzione ad elementi quali la solidarietà tra i membri della comunità o la struttura politico-formale di controllo del territorio. E però questi vantaggi sono ottenuti al prezzo di un sostanziale svuotamento del concetto di identità collettiva, che viene trasfigurata in termini di caratteri socio-strutturali di una comunità. Ciò purtroppo ostacola la spiegazione del fenomeno della secessione sul versante dell'azione (politica) collettiva - al quale non possiamo in verità rinunciare, come vedremo in seguito ${ }^{30}$.

La seconda direzione di risposta all'interrogativo «chi è il gruppo titolare del diritto di secessione?» consente di ovviare ai limiti denunciati per la prima. Essa, grazie al concetto di «attore collettivo», riesce più coerentemente a ritrarre una comunità in termini di identità collettiva. Il punto qualificante è l'attribuzione del diritto (di gruppo) alla secessione a quel gruppo che lo reclama esplicitamente, attraverso atteggiamenti e comportamenti, salvo naturalmente verificarne la legittimità sulla base degli argomenti morali pro e contro - poiché la legittimità della secessione resta condizionata.

$\mathrm{Ma}$ anche questo approccio incontra suoi specifici e numerosi problemi. Intanto, ad esempio, corre il rischio di far perdere di vista quel criterio identificante così essenziale che è il territorio, ben focalizzato invece dal concetto socio-strutturale di comunità visto sopra. In quanto attore collettivo, il gruppo cui attribuire il diritto di secessione deve poter fare riferimento ad una sua base territoriale, dato che «si secede con un territorio». $\mathrm{Ma}$ con il concetto di attore collettivo non siamo in grado di circoscrivere la parte di territorio per la quale è in questione la secessione. Il concetto di attore collettivo ci consente di cogliere che un gruppo reclama la secessione, si organizza, passa all'azione, ma di per sé non ci dice a quale unità socio-territoriale possa applicarsi il diritto a secedere; lascia nell'ombra, insomma, i criteri e $\mathrm{i}$ confini che identificano la comunità che vuole separarsi da quella comunità più ampia che la ricomprende - lo Stato-nazione. Anche nel caso più estremo (che svela in ultimo

30 Del resto lo stesso Tilly (1976) non ha esitazioni a dire che un approccio sociostrutturale - da solo - si rivela alla fine inadeguato quando si tratta di spiegare un fenomeno politico, quale ad esempio, nel suo caso, la controrivoluzione vandeana. 
la natura squisitamente politica della secessione), in cui si ritenga che possa/debba essere l'attore collettivo stesso che reclama il diritto di secedere come gruppo ad indicare/proporre i criteri e confini della comunità secessionista, si tratterà pur sempre di circoscrivere una comunità col suo territorio. Del resto è solo su queste basi che si potrà verificare la legittimità della pretesa secessionista, non fosse altro che per orientarsi in casi-limite, inverosimili quando si immagina un centinaio di condomini di alcuni caseggiati che, in quanto attore collettivo, reclamano la secessione - ma meno inverosimili in altri casi. Pensiamo, anche solo per un momento, al secessionismo leghista e al problema della delimitazionne socio-territoriale della «Padania».

Una teoria della secessione che voglia identificare l'unità e il soggetto cui riferire il diritto (di gruppo) di secessione incontra dunque ostacoli quando procede con approcci alternativamente centrati sul concetto di comunità o su quello di attore collettivo. Qui mi basta suggerire che la via d'uscita va probabilmente ricercata in una formulazione del concetto di identità collettiva che contemperi e saldi insieme quella dimensione «strutturale» del «territorio sociale» focalizzata come «comunità» e quella «volontaristica» dell' «azione intenzionale» focalizzata nei termini di «attore collettivo» ${ }^{31}$.

Questo tipo di impostazione concettuale va tenuto presente anche su un altro versante, quello degli argomenti/ragioni morali pro-secessione, il quale presenta sue proprie difficoltà. Infatti, diversamente da quanto lascerebbe supporre il discorso normativo, il fatto che filosofi politici elaborino argomenti morali favorevoli (o contrari) alla secessione non significa ancora che questi siano operativi nell'orientare le dispute secessioniste nei contesti concreti. Né è in grado di determinare, sullo sfondo della natura variabile dei contenuti degli argomenti morali sopra evidenziati, quali siano quelli più idonei a definire la si-

31 Integrare approcci basati su teorie strutturali ed approcci basati su teorie dell'azione è un problema che da sempre impegna le scienze sociali, dalla loro nascita con Weber e Durkheim (ma anche Marx) fino ad oggi con gli studi, ad esempio, del processo decisionale o con contributi quali quelli di Berger e Luckmann (1969); Almond e Powell (1988); Crozier e Friedberg (1978); Giddens (1984). Su questo punto rinvio a Nevola (1989a). Nel campo dello studio specifico della secessione tra i contributi teorici più avanzati e articolati è certamente Hechter (1992), nel quale si propone uno schema analitico di tipo dinamico basato su quattro stadi: 1) identificazione del gruppo regionale; 2) azione collettiva del gruppo regionale; 3 ) basi sociali del movimento secessionista; 4) reazione dello Stato che «ospita» la regione secessionista. Si veda anche Hechter (1979). 
tuazione delle pretese secessioniste (o anti-secessioniste) in una data circostanza. E come se tali argomenti non avessero una loro vera e propria «esistenza sociale» capace di incidere effettivamente sulle condotte pubbliche. Per meglio dire, è come se tali argomenti si trovassero in una condizione di «latenza»: esistono, invero, possono essere utilizzati e quindi influire in qualche modo sulle vicende collettive, ma restano appunto «argomenti potenziali» fin quando non vengono attivati. Ovvero, fin quando un soggetto capace di azione collettiva non li utilizza come motivi e strumenti in virtù dei quali definire la propria identità, le ragioni della propria esistenza e ricerca di affermazione, gli scopi da raggiungere. Segue, come corollario, che del repertorio di argomenti morali potenzialmente disponibili, in circostanze date e col variare delle circostanze, alcuni verranno attivati, altri no, e altri ancora potranno essere «inventati», costruiti o rimodellati - volta per volta, contesto per contesto.

Data la natura, in ultimo politica, dell'istanza secessionista, l'attore collettivo alle prese con l'«attivazione» di argomenti normativi assume l'aspetto di un vero e proprio imprenditore politico, che nella fattispecie si occupa di secessione. Da un'angolatura appena un po' diversa, possiamo dire che l'attivazione di argomenti normativi latenti coincide con un'operazione di «politicizzazione». Quest'ultima, peraltro, coincide anche con una selezione di rilevanza di alcuni argomenti morali piuttosto che altri, trafficando volta a volta col repertorio di argomenti morali potenziali e con la loro natura variabile (culturale, politico-territoriale, economica, storica). Gli argomenti morali abbandonano la loro condizione di «esistenza sociale» latente quando di fatto vengono politicizzati, assumendo così peso nell'orientare i termini del dibattito pubblico e valenza di risorsa per l'azione politica. «Politicizzazione» significa, chiaramente, che un tema acquista rilievo politico ${ }^{32}$. Esso partecipa, cioè, di quell'arena di conflitto e di governo del conflitto la cui posta in gioco - nei casi ordinari - è la distribuzione di autorità e di identità capaci di vincolare i comportamenti pubblici. Ma la cui posta

32 Cfr. Schmitt (1972). Un esempio di come in una situazione conflittuale concreta, contrasti sociali di diversa natura (economici, religiosi, amministrativi) si «politicizzano» e danno vita a conflitti propriamente politici è rinvenibile nell'analisi della controrivoluzione vandeana di Tilly (1976). Più in generale si veda anche Tilly (1978). Da parte sua, nella sua analisi delle fratture socio-territoriali, anche Rokkan (1982) ha messo in risalto l'importanza della loro politicizzazione. Vedi anche Rokkan e Urwin (1982). 
in gioco in casi «eccezionali» può diventare la stessa legittimità delle procedure e dei vincoli che regolano la distribuzione di autorità e di identità e quindi, come è con la secessione, la stessa legittimità di quell'ordinamento autoritativo-identitario che è lo Stato-nazione.

La capacità di attivare gli argomenti morali, la politicizzazione che rende loro piena esistenza sociale, non dipende esclusivamente e forse nemmeno prioritariamente dalla loro «bontà», da una loro qualità morale intrinseca. Essa ha probabilmente più a che vedere con la «razionalità» politica dell'imprenditore politico, con la sua abilità nel porre al centro dell'attenzione pubblica l'istanza secessionista e gli argomenti morali atti a legittimarla, con il suo successo come imprenditore del consenso pro-secessionista. Con questo però non intendo sostenere che gli argomenti morali in quanto tali siano del tutto «inesistenti», o che essi e la loro qualità/persuasività morale siano da ritenere meramente ininfluenti. $\mathrm{O}$, ancora e soprattutto, che il contesto sociopolitico in cui l'imprenditore politico muove la sua azione vada considerato immune, estraneo a motivazioni secessioniste, che sia del tutto insensibile a queste, e che dunque il successo che esso può tributare all'imprenditore secessionista sia frutto esclusivo della persuasione/forzatura prodotte dalla capacità di manipolazione sociopolitica di quest'ultimo. Il quadro concettuale esplicativo resta quello cui ho accennato in precedenza: l'attivazione (politicizzazione) degli argomenti morali e il credito cui può andare incontro un'istanza secessionista dipendono dal formarsi di un'identità collettiva improntata alla causa o alla prospettiva secessionista. $\mathrm{Ma}$ anche in questo caso, l'identità collettiva matura col concorso e col congiungersi della (volontaristica) capacità di azione dell'attore collettivo e delle (strutturali) caratteristiche socio-territoriali della comunità ${ }^{33}$. La seconda dimensione funge da premessa per la prima. Ma la sua valenza resta,

33 Cfr. Hechter (1992), sebbene il quadro esplicativo elaborato dall'autore non paia del tutto immune da propensioni di tipo struttural-deterministico di fronte al problema dell'azione collettiva. Per inciso, ritengo più persuasiva un'analisi dei fenomeni storico-sociali e politici ispirata alla concezione «contingentista» weberiana. Si veda ad esempio Weber (1974). A una concezione di questo tipo è legato l'approccio strategicocoercitivo al conflitto politico; cfr. Nevola (1994). Un'inclinazione esplicativa di tipo struttural-deterministico è alla base, ad esempio, di Skocpol (1981); una di segno contrario e più orientata a cogliere le ragioni del perché un'azione collettiva si verifichi oppure no, si sviluppi in un certo modo piuttosto che in un altro, a parità di condizioni di contesto, è ad esempio in Tarrow (1990). 
per così dire, inespressa $o$, appunto, latente, fin quando non entra in gioco la prima dimensione. In ultimo, i caratteri della comunità socio-territoriale possono vincolare i margini d'azione dell'attore collettivo, fino a predefinire un determinato repertorio di tematiche, di argomenti, di azioni collettive - che può anche escludere l'istanza secessionista. Ma l'attore collettivo può ridefinire tale repertorio, può «reinventare» i caratteri della comunità, decostruendo e ricostruendo sulla base di elementi di contesto, disponibili nella comunità di riferimento in quanto socialmente percepiti e diffusi - fino a ricomporne i tratti di identità collettiva propensa alla secessione, o tentarne un ritratto da usare come «offerta politica» capace di suscitare adesione, identificazione. Il secessionismo nostrano leghista viene subito alla mente, e quindi va analizzato anche da questa angolatura, come del resto gli altri casi di secessionismo nel mondo.

Il problema della rappresentanza e il soggetto politico della secessione

La secessione come diritto di gruppo presuppone, in fondo, una sorta di «coincidenza» tra l'imprenditore politico e la sua comunità di riferimento, quella per la quale rivendica la secessione. $\mathrm{O}$ meglio: solleva quello che possiamo definire il «problema della rappresentanza politica». Il problema nasce dal fatto che l'attore collettivo «attivo» sarà inevitabilmente più «ristretto» del gruppo-comunità per il quale avanza istanze secessioniste. Mentre dobbiamo presupporre una qualche identificazione tra tale attore e i membri della comunità territoriale, il grado specifico di identificazione è tutt'altro che scontato e non è facilmente accertabile. La conseguenza di questa situazione è che l'identificazione tra il soggetto politico e la sua comunità di riferimento tende spesso a diventare essa stessa una posta in gioco nelle contese secessioniste. Per quanto si possa riconoscere all'imprenditore della secessione una capacità di mobilitazione alla causa secessionista, resta che il concetto di attore collettivo designa un soggetto organizzato (un movimento, un partito) che definisce scopi e agisce intenzionalmente in nome di una collettività più ampia. In base ai suoi requisiti tipici, un attore collettivo può essere, quindi, identificabile sul piano empirico. $\mathrm{Ma}$ anche su quello delle pretese normative in gioco, dato che l'attore collettivo organizzato, per così dire, «parla», espri- 
me le sue rivendicazioni con dichiarazioni e con iniziative. Non possiamo invece dire altrettanto per la comunità territoriale cui l'imprenditore secessionista fa riferimento. È ciò che crea difficoltà quando si tratta di considerare chi sia il «gruppo» (popolazione e suo insediamento territoriale) in nome del quale l'imprenditore politico reclama la secessione come «diritto di gruppo» - ma, ovviamente, non chiede che questo valga solo per $\mathrm{i}$ membri della sua organizzazione. Qui sta la rilevanza del problema della rappresentanza. Sia per il discorso normativo che per l'analisi politica fattuale.

Sul piano normativo, intanto, come facciamo a sapere in nome di chi parla legittimamente l'imprenditore politico della secessione? Esprime questo effettivamente e legittimamente la volontà della comunità territoriale cui dice di riferirsi? Per affrontare interrogativi di questa portata il discorso normativo non può fare a meno di agganciare gli argomenti morali al contesto politico-istituzionale in cui cade la rivendicazione secessionista, recuperando le «ragioni procedurali» che legittimano un sistema democratico. Così, la verifica delle pretese di rappresentanza socio-territoriale riscopre, accanto agli argomenti morali e ai diritti di gruppo, argomenti più nitidamente «liberali», quali il principio di maggioranza e il meccanismo democratico-procedurale del voto - e quindi il diritto individuale sul quale questi si fondano ${ }^{34}$.

Non intendo qui entrare nel merito dei dettagli delle soluzioni normative cui consentirebbe di pervenire una ripresa di criteri procedurali di verifica e di legittimazione dettata dalla necessità di chiarire l'identificazione tra imprenditore politico della secessione e la comunità territoriale cui questo fa riferimento, se e quanto per il primo è data la rappresentanza della seconda $a^{35}$. Mi limito a sottolineare che la situazione più semplice, forse più diffusa e comunque più richiamata nel dibattito normativo, prevede un contesto politico-istituzionale dove la forma dello Stato è di tipo federale. Anche sotto questo riguardo, il caso paradigmatico è quello del Canada e delle rivendicazioni secessioniste del Quebec ${ }^{36}$. In effetti, la struttura federale

34 Cfr. ad esempio Buchanan (1994). Sotto un profilo non normativo ma empirico-esplicativo anche Dion (1996).

35 Il punto trova ampia trattazione, anche sul piano della costituzionalizzazione del diritto di secessione, in Buchanan (1994).

$36 \mathrm{E}$ anche utile tenere presente, ad esempio, il caso catalano o quello basco in Spagna. Cfr. ad esempio Bachoud (1993); Clark (1987). 
dello Stato rende disponibile l'esistenza di sedi di rappresentanza politico-istituzionale delle varie comunità socio-territoriali. In un quadro del genere, per l'imprenditore politico della secessione è chiarito il canale di verifica della legittimità delle sue istanze a nome di un'intera collettività: l'organo assembleare dello Stato federato membro del più ampio sistema federale. Qui le procedure liberaldemocratiche possono consentire di misurare la rappresentanza effettiva del soggetto politico che reclama la secessione, secondo un preciso ed istituzionalizzato ritaglio territoriale-elettorale. Non solo: sono anche dati un contesto e un vincolo politico-istituzionali alla lotta secessionista. Un quadro, questo, che facilita anche, eventualmente, le modalità di ricorso allo strumento referendario come meccanismo di verifica delle pretese secessioniste di un soggetto politico e il loro grado di rappresentanza della «volontà» della comunità socio-territoriale. In definitiva, una struttura politica di tipo federale consentirebbe di sciogliere i nodi relativi all'accertamento della titolarità di un diritto di gruppo sulla base di principi e dispositivi tipicamente liberaldemocratici e procedurali. Così come essa sembrerebbe, più sottilmente, mettere un argine alle difficoltà, se non all'indeterminatezza, di un discorso normativo improntato solo su argomenti morali.

La forza di questa soluzione è tuttavia meno dirimente di quanto sembri a prima vista. Tale impostazione normativa apre certamente prospettive per un trattamento dei nostri problemi, ma solo entro alcune e specifiche situazioni. Li lascia invece al punto in cui li incontra in molti altri casi, forse nella maggior parte. Da un lato, anche all'interno di una forma statale di tipo federale. E ciò quando incontriamo istanze secessioniste espresse da imprenditori politici che si dicono agire in nome di una comunità socio-territoriale alla quale l'ordinamento federale dello Stato non ha fornito alcun ritaglio politico-istituzionale ed elettorale proprio, il quale possa costituirsi come sede e canale di verifica della rappresentanza del soggetto collettivo reclamante, secondo procedure liberaldemocratiche. Come cogliere, in casi del genere, quella sorta di identificazione tra richieste dell'attore collettivo organizzato e la «sua» comunità socio-territoriale, sulla base della quale il discorso normativo acconsente alla titolarità del diritto (di gruppo) alla secessione? Ci ritroviamo al punto di partenza.

Dall'altro lato, l'impostazione normativa proceduralista tace completamente in tutte le situazioni di tipo non-federale. Natu- 
ralmente, anche in questi casi il discorso normativo incontra il problema del gruppo titolare del diritto di secessione. E difatti è costretto ad ammettere che il ricorso a soluzioni improntate ad un normativismo liberal-procedurale non è sufficiente a chiarire la questione in gioco. La tendenza prevalente nel dibattito morale è, implicitamente o esplicitamente, quella di ritornare a sottolineare il criterio degli argomenti morali - e, però, con i silenzi e le irrisolutezze che ritornano tali e quali li abbiamo visti sopra.

In verità, dalle ultime considerazioni fatte si intravede un punto di uscita per le difficoltà in cui il discorso normativo viene a cadere quando si tratta di mettere in luce qual è la collettività cui può andare la titolarità del diritto di secessione. Tale punto di uscita rimanda ad una sorta di raccordo tra ordinamento federale e diritto di secessione come diritto di gruppo, là dove l'istanza del federalismo tende a costituirsi come premessa per una (successiva) rivendicazione del diritto a secedere. Il punto resta comunque controverso di suo, non fosse altro per il fatto che le costituzioni democratiche (anche quelle federali), per dirne una, non sono poi così solidali col diritto di secessione, né, tanto meno, prevedono specifici dispositivi al riguardo. Non è un caso che la politica della secessione, quando vuole essere coerente con se stessa, preferisce mettere in circolazione la prospettiva del confederalismo - questa sì compatibile con istanze secessioniste. In questo senso, quando il linguaggio politico del leghismo nostrano offre il confederalismo come alternativa al secessionismo in realtà mantiene salde le sue pretese secessioniste, sia pure inquadrandole in un percorso di radicale cambiamento istituzionale della forma dello Stato-nazione italiano. Il rapporto tra secessionismo, federalismo e confederalismo è, in effetti, un altro importante capitolo che attiene alla teoria della secessione. $\mathrm{Ma}$ in questa sede ci porterebbe troppo lontano. Meglio procedere oltre, dopo averlo richiamato ${ }^{37}$.

Di fronte al problema della rappresentanza che accompagna la secessione come diritto di gruppo rimane il sostanziale imbarazzo del discorso normativo - sia quando questo si concentra esclusivamente sugli argomenti morali, sia quando si apre a soluzioni improntate a criteri di tipo procedurale. Le difficoltà di

37 In questi ultimi anni Linz (1995; 1997) sta indagando sul rapporto complesso che intercorre tra Stato-nazione, regime democratico e prospettive istituzionali federaliste, in particolare nel contesto di società multietniche o multiculturali. 
un'analisi esclusivamente normativa del «chi rappresenta chi» sono un'altra spia dello statuto essenzialmente politico della secessione. Ciò che a questo punto diventa necessario è il passaggio da un'analisi normativa ed etico-politica ad una empirica e realistico-politica, senza che questo significhi minimizzare l'importanza della prima in se stessa e per il peso che essa conserva anche nella seconda.

In ultimo, al problema della rappresentanza, del «chi rappresenta chi», e di chi, dunque, è titolare del diritto di secessione, la risposta è: chi riesce a farsi «riconoscere» come tale - ossia, come imprenditore della secessione. Ma farsi «riconoscere» da chi?, come? Intanto, dalla comunità socio-territoriale nel nome della quale il soggetto politico organizzato dichiara di parlare $^{38}$. E questo ripropone l'esigenza di analisi delle dinamiche socio-politiche che interessano, attraverso un raccordo tra $\mathrm{i}$ concetti di attore collettivo e di comunità socio-territoriale. Un raccordo che richiede un approfondimento a sé stante e che qui lascio in sospeso con le scarne indicazioni di prospettiva più sopra formulate. $\mathrm{Al}$ momento, infatti, mi interessa mettere in risalto un secondo modo in cui l'imprenditore della secessione si fa «riconoscere» come tale e quindi come titolare di pretese in ordine al diritto di secessione. In questo caso «farsi riconoscere» significa farsi riconoscere come soggetto politico, ossia come soggetto che agisce nella sfera politica, nell'ambito della quale intrattiene relazioni di potere competitive e conflittuali con altri soggetti politici, sulla base della propria identità e pretese secessioniste e in vista della distribuzione dell'autorità.

È pressoché inevitabile che questo «riconoscimento politico» si realizzi sul piano di quella che abbiamo chiamato «politica ordinaria», e quindi secondo canali e modalità tipici della democrazia: competizione tra partiti, consenso elettorale, conflitti per la distribuzione dell'autorità costituita nel rispetto di vincoli e procedure costituzionali - i quali implicano, innanzitutto, monopolio statale dell'uso legittimo della forza, patto democratico tra le parti politiche e unitarietà territoriale del sistema d'autorità con validità erga omnes. Tuttavia, nel caso della secessione, il riconoscimento politico comporta, altrettanto inevitabilmente, il passaggio dalla politica ordinaria alla «politica eccezionale», quella in cui non è già definito $\mathrm{ma}$ in cui invece si

38 È questa - come già sopra rilevato - la strada seguita da Hechter (1992). 
decide «chi decide dello e nello stato di crisi», ossia quando viene a mancare il riconoscimento, condiviso tra le parti, del sistema d'autorità costituito, che è poi quello incarnato dallo Stato. A differenza di quanto preveda la politica ordinaria, col passaggio alla politica extra-costituzionale i canali e le modalità di riconoscimento politico dell'imprenditore della secessione richiamano non già o non tanto la competizione elettorale con gli altri partiti del sistema democratico, ma un conflitto con lo Stato-nazione stesso, la cui posta in gioco è la legittimità del monopolio dell'autorità, pretesa dallo Stato ma non riconosciuta e sfidata dall'imprenditore della secessione. Un po' in analogia col quadro politico del sistema internazionale classico, il conflitto tra le parti diventa un conflitto tra soggetti con pretese di sovranità (politico-territoriale) esclusiva e indivisibile. Insomma, ad una situazione di monopolio e unitarietà della sovranità subentra una di «sovranità multipla», o caratterizzata da pretese multiple di sovranità - esattamente come è tipico delle situazioni rivoluzionarie o di guerra civile.

Una situazione di quest'ultimo tipo si verifica, e quindi l'imprenditore della secessione consegue un riconoscimento in quanto tale e di tipo puramente politico, in base a due condizioni essenziali. La prima, per così dire preliminare, è che l'imprenditore della secessione riesca ad introdurre nel sistema politico democratico un cleavage, una linea di frattura tra le forze politiche che divida tra «unitaristi» e «secessionisti» ${ }^{39}$. La seconda condizione, cruciale, è che l'opzione «uscita» dall'unità politico-territoriale costituzionalizzata si riveli (o sia ritenuta) una risorsa in grado di infliggere danni politici, secondo due principali direzioni: 1) indebolimento del consenso politicoelettorale degli altri soggetti politici, quelli «leali» all'ordinamento di autorità democratico costituito; 2) delegittimazione dell'ordinamento stesso e minaccia di destabilizzazione dell'ordine sociale e politico. Quando, sulla base di queste condizioni, le forze politiche «leali» ${ }^{40}$ e più ancora le istituzioni dello Stato

39 Questo cleavage potrebbe essere inteso come una riattualizzazione o riformulazione di quello socio-territoriale «centro vs. periferia» individuato da Rokkan (1982) come una delle fratture genetiche alla base della struttura dei sistemi politici e partitici degli Stati europei. Oppure come presa di coscienza politica da parte di un gruppo di una storia di «colonialismo interno» da superare, secondo l'interpretazione dei movimenti etnici e nazionalistici di Hechter (1979).

40 Per i concetti di forza politica «leale», «semi-leale» e «sleale» cfr. Linz (1981). 
competono per il consenso elettorale, trattano e negoziano decisioni politiche di qualsivoglia natura o contenuto con chi si propone come imprenditore della secessione, di fatto si è realizzato il riconoscimento di quest'ultimo come soggetto politico. Ovvero: questo ha avuto successo nel farsi riconoscere con la sua identità e le sue pretese secessioniste - indipendentemente dal fatto che le controparti politiche o le istituzioni dello Stato persistano (o meno) nel ritenere illegittima la secessione.

Qui sta, ancora una volta, la sostanza tutta politica del secessionismo. Il secessionista ha avuto successo. E riuscito, cioè, a politicizzare l'istanza di secessione. Non necessariamente in positivo, come istanza istituzionalizzata, ossia come questione in agenda nel processo politico democratico di riforma dell'ordinamento statuale-nazionale. Questa modalità può verificarsi e in talune circostanze si è verificata, come mostrano i casi esemplari, pur tra loro differenti, del Canada/Quebec o di secessione realizzata in modo «pacifico», quale quella della Norvegia dalla Svezia nel 1905 o quella che ha portato alla divisione della Cecoslovacchia nel 1993. Tuttavia la politicizzazione della secessione può aver luogo anche in chiave «negativa», nella forma non-istituzionalizzata di un conflitto politico centrato sull'issue della secessione. Per questa via trova realizzazione, contestualmente, quella politicizzazione delle ragioni morali della secessione, la quale traduce in argomenti influenti per la condotta politica quelli che altrimenti resterebbero argomenti socialmente esistenti ma politicamente latenti.

Sul doppio volto della politica della secessione. Una conclusione da cui ripartire

In effetti, come si capisce da quanto già osservato, se consideriamo le modalità di azione relative al riconoscimento dell'imprenditore della secessione come soggetto politico, notiamo che i piani della politica ordinaria costituzionalizzata e della politica «eccezionale» non-costituzionalizzata non restano separati ed incomunicanti tra loro, e alla fine, nei casi concreti, diventa talora difficile distinguere nitidamente i due. Da qui un'ambiguità o un'ambivalenza che risultano tipiche della politica della secessione in contesto democratico, e che segnano inesorabilmente le vicende e $\mathrm{i}$ comportamenti secessionisti. In proposito va tenuto presente che accanto alla via democratica e «pacifica» 
alla secessione, esiste la strada della lotta armata e violenta. In questa sede lascio da parte la seconda strada e le sue possibili interconnessioni con la prima, che non di rado si verificano nei casi concreti - basti pensare, per fare solo due esempi, alla questione basca o a quella irlandese. Mi preme comunque sottolineare che quest'ultimo è un aspetto di prima importanza, sul quale una teoria esplicativa ( $\mathrm{ma}$ anche normativa) non può tacere e che invece risulta piuttosto trascurato nel dibattito internazionale. Ma qui voglio limitarmi a considerare la via democratica, per una ragione precisa, che ha anch'essa a che fare con l'ambivalenza intrinseca della politica della secessione. La via democratica alla secessione, infatti, possiede un doppio volto: quello di politica ordinaria e quello di politica «eccezionale». Ciò che è qui interessante notare è che il secondo volto, anche quando ripulito da espressioni di violenza (fisica), svela il carattere non sempre risolutivo dei vincoli democratici per la politica della secessione ${ }^{41}$. Facciamo qualche passo in questa direzione, normalmente poco battuta negli studi in materia, ed invece di grande rilievo anche per capire alcuni aspetti del secessionismo leghista di casa nostra.

Andiamo con ordine. Intanto concludo su un punto: il riconoscimento dell'imprenditore della secessione come soggetto politico, quel riconoscimento che salda comportamenti e scopi dichiarati di un attore collettivo con la «sua» comunità socioterritoriale e che quindi consente di identificare il «gruppo» cui fare riferimento nel caso del diritto di gruppo di secessione, si realizza e si coglie, alla fine, sulla base di considerazioni attinenti anche alla capacità politica e non solo sulla base della pura qualità degli argomenti morali del secessionismo. Questa capacità politica dell'imprenditore della secessione si misura come capacità di ottenere successo nella politica ordinaria (consenso elettorale, presenza parlamentare, ruolo nel sistema dei partiti) in forza di una rivendicazione di politica «eccezionale» (sfida alla sovranità dello Stato-nazione, «uscita» dall'unità politica costituzionalizzata).

$\grave{E}$ chiaro che la riuscita di un'operazione politica di questo

41 Spunti interessanti su questo tema, che merita una specifica trattazione a sé, sono rinvenibili ad esempio in Linz (1981); Connor (1995), così come nell'ambito degli studi sui mutamenti di regime politico, per i quali, a titolo orientativo, cfr. Morlino (1980). Sul rapporto tra uso della forza e cambiamento politico, che qui sono costretto a tralasciare, mi si consenta intanto di limitarmi a rinviare a Nevola (1989b). 
tipo dipende dalla serietà della minaccia all'ordine costituito portata dal secessionismo, ossia da quanto l'imprenditore della secessione è in grado di infliggere danni (Nevola 1994) alle altre forze politiche democratiche e alle istituzioni dello Stato-nazione, ad esempio rendendosi indispensabile nel gioco delle alleanze tra le forze politiche, o per il funzionamento del processo decisionale politico ovvero portando alla delegittimazione dello Stato-nazione. Altrettanto chiaro è che questi meccanismi di riconoscimento politico dipendono altresì da come le altre parti politiche e le istituzioni dello Stato percepiscono e valutano la sfida secessionista e il suo imprenditore, da come li trattano e se trattano con l'una e con l'altro.

$\mathrm{Al}$ fondo, come tipicamente suggerisce un'analisi realistica dei fenomeni politici, il gioco del riconoscimento politico del secessionista è condizionato dalla «credibilità» della minaccia ${ }^{42}$ all'ordine costituito. In questa chiave, il fenomeno della secessione abbandona ogni riferimento esclusivo ad un quadro interpretativo dettato dagli argomenti morali o dal normativismo democratico (o liberaldemocratico) per situarsi in uno puramente politico, relativo al conflitto e al governo del conflitto che hanno come posta in gioco l'ordinamento stesso di autorità - e cioè la sovranità dello Stato-nazione. Siamo nel pieno di quel tipo di conflitto che chiamo extra-unit (Nevola 1994), ossia che sfugge al sistema di autorità unitario, vincolante le modalità di comportamento delle parti in lotta e da queste condiviso (Easton 1973). Un conflitto che è extra-unitario per il semplice fatto che almeno una delle parti (solitamente quella secessionista) si pone, per sua stessa identità politica, per pretese e comportamenti, fuori dal sistema unitario di autorità dello Stato-nazione, e ciò secondo condizioni tali da esigere un «trattamento politico» invece, o prima ancora, di uno di tipo giudiziario. Ciò naturalmente non vuol dire che lo Stato sfidato nella sua sovranità e unitarietà non sia tenuto o non tenti di definire la questione in termini giudiziari, di legalità o di costituzionalità, nel caso anche con l'applicazione della forza fisica - della quale detiene (e pretende) tipicamente il monopolio legittimo. Il fatto è, però, che la sfida secessionista colpisce per definizione i fondamenti di legittimità politica dello Stato, della sua sovranità/autorità e

42 Su questi aspetti dell'agire politico restano utili punti di riferimento Schelling (1968; 1977). Cfr. anche Rusconi (1984) e Nevola (1994). 
quindi della stessa pretesa dello Stato di definire la secessione sul piano della legalità costituita. È qui che risiede il carattere extra-unitario del conflitto secessionista, il suo statuto propriamente politico (e di «politica di eccezione») di cui ho parlato all'inizio. Ed è qui che entrano in gioco le dinamiche sottese alla credibilità della minaccia politica del secessionismo ed acquista rilievo fondamentale il riferimento ai «danni politici» che l'imprenditore della secessione è in grado di recare alle altre forze politiche, al funzionamento del processo democratico e alle istituzioni dello Stato-nazione. In politica, «colpire» l'avversario, «fargli male» è un modo tipico per farsi riconoscere. A volte anche per fare riconoscere pretese o diritti secessionisti, anche quando le loro condizioni di ammissibilità morale e normativa risultano quantomeno controverse nello specifico caso concreto. Come spesso accade in politica.

Per concludere, è appena il caso di sottolineare che in questa sede abbiamo posto alcune premesse e condizioni per l'analisi del successo o meno della secessione - successo ora inteso come esito concreto e politico-istituzionale di un conflitto secessionista. Per il resto, un'analisi di quest'ultimo aspetto resta da svolgere e non ha ancora trovato adeguata trattazione teorica negli studi sulla secessione. Un'analisi del genere dovrà: 1) prestare particolare attenzione alle modalità dinamiche e strategico-coercitive del conflitto tra attore politico secessionista (movimento, partito) e attore politico unitarista (Stato, altri partiti); 2) tenere conto delle complessive condizioni sociali di contesto e tradurle in vincoli e/o opportunità di azione politica; 3) approfondire quel rapporto complicato tra secessione e democrazia, caratterizzato, ad un tempo, da attrazione ed incompatibilità tra l'una e l'altra. Si tratta di un indirizzo di riflessione e di ricerca che sarà utile seguire per mettere a fuoco il fenomeno specifico della secessione e per risistemare gli strumenti dell'analisi socio-politica in vista di una comprensione di tendenze sempre più rilevanti nella politica dei nostri tempi.

\section{Riferimenti bibliografici}

AA.VV. (1995), Le nuove frontiere dell'America. Il caso del Québec, in «Limes», 3, pp. 197-293.

AA.VV. (1996), Disfare l'Italia per fare l'Europa?, in «Limes», 3, pp. 21-116. 
Almond, G.A. e G.B. Powell jr. (1988), Politica comparata. Sistema, processi e politiche, Bologna, Il Mulino.

Arendt, H. (1985), La disobbedienza civile, in H. Arendt, Politica e menzogna, Milano, SugarCo.

Bachoud, A. (1993), Il vento separatista della Catalogna, in «Limes», 4, pp. 207-218.

Bendix, R. (1969), Stato nazionale e integrazione di classe, Bari, Laterza.

Beran, H. (1984), A Liberal Theory of Secession, in «Political Studies», 1, pp. 21-31.

- (1988), More Theory of Secession, in «Political Studies», 2, pp. 316-323.

Berger, P.L. (1981), Le piramidi del sacrificio. Etica politica e trasformazione sociale, Torino, Einaudi.

Berger, P.L. e Th. Luckmann (1969), La realtà come costruzione sociale, Bologna, Il Mulino.

Bhalla, R.S. (1991), The Right of Self-Determination in International Law, in Twining (1991).

Biorcio, R. (1997), Padania promessa, Milano, Il Saggiatore.

Birch, A.H. (1984), Another Liberal Theory of Secession, in «Political Studies», XXXII, pp. 596-602.

Bonanate, L. (1994), I doveri degli Stati, Bari, Laterza.

Bookman, M.Z. (1993), The Economics of Secession, London, Macmillan.

Brilmayer, L. (1991), Secession and Self-Determination: A Territorial Interpretation, in «Yale Journal of International Law», 1, pp. 177 202.

Buchanan, A. (1994), Secessione. Quando e perché un paese ba il diritto di dividersi, Milano, Mondadori.

- (1997) Theories of Secession, in «Philosophy \& Public Affairs», 1, pp. 31-61.

Bucheit, L.C. (1978), Secession: The Legitimacy of Self-Determination, New Haven, Yale University Press.

Buttino, M. e G. Rutto (a cura di) (1997), Nazionalismi e conflitti etnici nell'Europa orientale, Milano, Feltrinelli.

Cartocci, R. (1994), Fra Lega e Chiesa. L'Italia in cerca di integrazione, Bologna, il Mulino.

Cassese, A. (1984), Il diritto internazionale nel mondo contemporaneo, Bologna, il Mulino.

Clark, R.P. (1987), 'Rejectionist' Voting as an Indicator of Ethnic Nationalism: the Case of Spain's Basque Provinces, 1976-1986, in «Ethnic and Racial Studies», 4, pp. 427-447.

Chapman, J.W. e I. Shapiro (a cura di) (1993), Democratic Community, New York, New York University Press.

Connor, W. (1995), Etnonazionalismo. Quando e perché emergono le nazioni, Bari, Dedalo. 
Crozier, M. e E. Friedberg (1978), Attore sociale e sistema. Sociologia dell'azione organizzata, Milano, Etas.

Cuaz, M. (1997), L'identità ambigua: l'idea di nazione tra storiografia e politica, in «Rivista Storica Italiana», in corso di pubblicazione.

Dahl, R.A. (1986), Poliarchia. Partecipazione e opposizione nei sistemi politici, Milano, Angeli.

De Fiores, C. e D. Petrosino (1996), Secessione, Roma, Ediesse.

De-Shalit, A. (1996), National Self-Determination: Political, not Cultural, in «Political Studies», 5, pp. 906-920.

Diamanti, I. (1995), L'improbabile ma rischiosa secessione, in «il Mulino», 5, pp. 811-820.

- (1996a), Il Nord senza l'Italia, in «Limes», 1, pp. 15-30.

- (1996b), Il male del Nord. Lega, localismo, secessione, Roma, Donzelli.

Dion, S. (1996), Why is Secession Difficult in Well-Established Democracies? Lessons from Quebec, in «British Journal of Political Science», 2, pp. 269-283.

Easton, D. (1973), Il sistema politico, Milano, Comunità.

Ferrajoli, L. (1997), La sovranità nel mondo moderno, Bari, Laterza.

Ferrara, A. (a cura di) (1992), Comunitarismo e liberalismo, Roma, Editori Riuniti.

Finer, S.E. (1974), State-Building, State Boundaries and Border Control, in «Social Science Information», XIII, pp. 79-126.

Freund, J. (1983), Sociologie du conflit, Paris, Presses Universitaires de France.

- (1995), Il terzo, il nemico, il conflitto. Materiali per una teoria del Politico, Milano, Giuffrè.

Gamson, W.A. (1968), Power and Discontent, Homewood, Dorsey Press.

Giddens, A. (1984), The Constitution of Society, Oxford, Blackwell.

Gurr, T.R. (1970), Why Men Rebel, Princeton, Princeton University Press.

Habermas, J. (1985), Etica del discorso, Bari, Laterza.

- (1996), Fatti e norme. Contributi a una teoria discorsiva del diritto e della democrazia, Milano, Guerini e Associati.

Hampshire, S. (1995), Innocenza ed esperienza. Un'etica del conflitto, Milano, Feltrinelli.

Hechter, M. (1979), Il colonialismo interno. Il conflitto etnico in Gran Bretagna, Scozia, Galles e Irlanda: 1536-1966, Torino, Rosenberg \& Sellier.

- (1992), The Dynamics of Secession, in «Acta Sociologica», 4, pp. 267-283.

Hirschman, A.O. (1982), Lealtà, defezione, protesta. Rimedi alla crisi delle imprese, dei partiti e dello stato, Milano, Bompiani.

Horowitz, D.L. (1985), Ethnic Groups in Conflict, Berkeley, University of California Press. 
Hume, D. (1987), Dei partiti in generale, in Opere filosofiche, vol. III, Bari, Laterza.

Kelsen, H. (1994), Teoria generale del diritto e dello Stato, Milano, Etas.

Kymlicka, W. (1995), Multicultural Citizenship, Oxford, Clarendon Press.

Lasswell, H.D. e A. Kaplan (1979), Potere e società. Uno schema concettuale per la ricerca politica, Milano, Etas.

Linz, J.J. (1981), Il crollo dei regimi democratici: un modello teorico, in J.J. Linz, P. Farneti e M.R. Lepsius, La caduta dei regimi democratici, Bologna, Il Mulino.

- (1995), Plurinazionalismo e democrazia, in «Rivista Italiana di Scienza Politica», 1, pp. 21-50.

- (1997), Democracy Today: An Agenda for Students of Democracy, in «Scandinavian Political Studies», 2, pp. 115-134.

Luhmann, N. (1969), Legitimation durch Verfabren, Berlin, Neuwied.

Matteucci, N. (1993), Lo Stato moderno. Lessico e percorsi, Bologna, Il Mulino.

McIlwain, C.H. (1990), Costituzionalismo antico e moderno, Bologna, Il Mulino.

Melucci, A. (a cura di) (1976), Movimenti di rivolta. Teorie e forme dell'azione collettiva, Milano, Etas.

Melucci, A. e M. Diani (1992), Nazioni senza stato. I movimenti etnico-nazionali in Occidente, Milano, Feltrinelli.

Michalska, A. (1991), Rights of Peoples to Self-determination in International Law, in Twining (1991).

Miglio, G. (1993), Disobbedienza civile, Milano, Mondadori.

Miglio, G. e A. Barbera (1997), Federalismo e secessione. Un dialogo, Milano, Mondadori.

Montesquieu (1989), Lo spirito delle leggi, Milano, Rizzoli.

Morlino, L. (1980), Come cambiano i regimi politici. Strumenti di analisi, Milano, Angeli.

Nevola, G. (1989a), Processo decisionale e guerra. Un approccio integrato, in «Quaderni di Sociologia», 12, pp. 99-133.

- (1989b), Una politica radicale per il cambiamento e il problema dell'uso della forza, in «Rassegna Italiana di Sociologia», 1, pp. 117129.

- (1994), Conflitto e coercizione. Modello di analisi e studio di casi, Bologna, Il Mulino.

- (1997), Il ritorno della nazione, in «Il Politico», 1, pp. 143-161.

Nielsen, K. (1993), Secession: The Case of Quebec, in «Journal of Applied Philosophy», 10, pp. 29-44.

Nordlinger, E.A. (1972), Conflict Regulation in Divided Societies, Harvard, Center for International Affairs Harvard University.

Poggi, G. (1978), La vicenda dello Stato moderno. Profilo sociologico, Bologna, Il Mulino. 
Rampini, F. (1996), Conviene alla Padania la secessione?, in «Limes», 1, pp. 31-36.

Rokkan, S. (1982), Cittadini, elezioni, partiti, Bologna, Il Mulino.

Rokkan, S. e D. Urwin (a cura di) (1982), The Politics of Territorial Identity, London, Sage.

Rosanvallon, P. (1994), La rivoluzione dell'uguaglianza. Storia del suffragio universale in Francia, Milano, Anabasi.

Rumiz, P. (1997), La secessione leggera, Roma, Editori Riuniti.

Rusconi, G.E. (1984), Scambio, minaccia, decisione. Elementi di sociologia politica, Bologna, Il Mulino.

- (1993), Se cessiamo di essere una nazione, Bologna, Il Mulino.

- (1994), L'identità nazionale e la sfida separatista, in G. Spadolini (a cura di), Nazione e nazionalità in Italia, Bari, Laterza.

- (1997), Patria e repubblica, Bologna, Il Mulino.

Sartori, G. (1993), Democrazia. Cosa è, Milano, Rizzoli.

Schelling, Th. (1968), La diplomazia della violenza, Bologna, Il Mulino.

- (1977), The Strategy of Conflict, Oxford, Oxford University Press.

Schmitt, C. (1972), Il concetto di 'politico', in Id., Le categorie del 'politico', Bologna, Il Mulino.

Schnur, R. (1979), Individualismo e assolutismo, Milano, Giuffrè.

Skocpol, T. (1981), Stati e rivoluzioni sociali. Un'analisi comparata di Francia, Russia e Cina, Bologna, Il Mulino.

Smith, A.D. (1984), Il revival etnico, Bologna, Il Mulino.

Stuart Mill, J. (1997), Considerazioni sul governo rappresentativo, Roma, Editori Riuniti.

Tamir, Y. (1993), Liberal Nationalism, Princeton, Princeton University Press.

Tarrow, S. (1990), Democrazia e disordine. Movimenti di protesta e politica in Italia. 1965-1975, Bari, Laterza.

Taylor, Ch. (1993), Multiculturalismo. La politica del riconoscimento, Milano, Anabasi.

Thoreau, H.D. (1993), Disobbedienza civile, Milano, Mondadori.

Tilly, Ch. (1976), La Vandea, Torino, Rosenberg \& Sellier.

- (1978), From Mobilization to Revolution, Reading, Addison-Wesley.

Tocqueville, A. de (1968), La democrazia in America, in A. de Tocqueville, Scritti politici, Torino, Utet.

Toso, F. (1996), Frammenti d'Europa. Guida alle minoranze etnico-linguistiche e ai fermenti autonomisti, Milano, Baldini \& Castoldi.

Twining, W. (a cura di) (1991), Issues of Self-Determination, Aberdeen, Aberdeen University Press.

Walzer, M. (1984), Liberalism and the Art of Separation, in «Political Theory», XII, pp. 315-329.

- (1987), Interpretation and Social Criticism, Cambridge, Harvard University Press. 
- (1990a), Guerre giuste e ingiuste. Un discorso morale con esemplificazioni storiche, Napoli, Liguori.

- (1990b), The Communitarian Critique of Liberalism, in «Political Theory», 1, pp. 6-23.

- (1991), La rinascita delle tribù, in «MicroMega», 5, pp. 99-111.

Weber, M. (1974), Studi critici intorno alla logica delle scienze della cultura, in Id., Il metodo delle scienze storico-sociali, Torino, Einaudi.

Zagrebelsky, G. (1992), Il diritto mite. Legge, diritti, giustizia, Torino, Einaudi. 